\title{
Cognitive behaviour therapy in chronic fatigue syndrome
}

\author{
S Butler, T Chalder, M Ron, S Wessely
}

\begin{abstract}
Fifty patients fulfilling operational criteria for the chronic fatigue syndrome (CFS), and who had been ill for a mean of five years, were offered cognitive behaviour therapy in an open trial. Those fulfilling operational criteria for depressive illness were also offered tricyclic antidepressants. The rationale was that a distinction be drawn between factors that precipitate the illness and those that perpetuate it. Among the latter are cognitive factors such as the belief that physical symptoms always imply tissue damage, and behavioural factors such as persistent avoidance of activities associated with an increase in symptoms. Therapy led to substantial improvements in overall disability, fatigue, somatic and psychiatric symptoms. The principal problems encountered were a high refusal rate and difficulties in treating affective disorders. Outcome depended more on the strength of the initial attribution of symptoms to exclusively physical causes, and was not influenced by length of illness. These results suggest that current views on both treatment and prognosis in CFS are unnecessarily pessimistic. It is also suggested that advice currently offered to chronic patients, to avoid physical and mental activity, is counterproductive.
\end{abstract}

The chronic fatigue syndrome, also known as postviral fatigue or myalgic encephalomyelitis, continues to attract attention and controversy. The scale of the problem is unknown, largely due to difficulties in case definition, ${ }^{1}$ but it seems probable that increasing numbers of people are being diagnosed, or diagnosing themselves, as suffering from the condition. At the beginning of 1989 over 150 people a week were joining the principal patients' organisation. ${ }^{2}$

Even nomenclature remains a problem in this field. Myalgic encephalomyelitis (ME) is frequently encountered, but is unsatisfactory for many reasons. ${ }^{3}$ Postviral fatigue syndrome implies an aetiology that is by no means certain. For the rest of this paper we have followed the international consensus of using the term "chronic fatigue syndrome" (CFS), as it is short, accurate and has no aetiological implications. ${ }^{4}$

The cause of CFS remains unknown.
Various factors, both medical and psychiatric, have been implicated. ${ }^{3}$ At present, however, evidence has only been presented from small, selected case control studies. ${ }^{1}$ Furthermore, such studies are only able to report associations, not aetiologies, and may not apply to the majority of patients with the clinical syndrome. $^{35}$ There are also no established treatments for CFS. Suggested methods of management that focus on presumed chronic infection have so far been found wanting. ${ }^{67}$ Instead, most patients receive very little in the way of practical help. The mainstay of treatment remains rest, and to wait either for remission or a medical cure, and the advice consistently offered by the current self help groups is to avoid physical and mental activity. Others are told to accept disability as "living within their limits". This state of affairs has been succinctly described as "therapeutic nihilism"8 and is unsatisfactory. What little we know about the natural history of these conditions ${ }^{9}$ shows that untreated they can be severe and persistent. In the largest current series "Most of the cases seen do not improve, give up their work and become permanent invalids". 6 No satisfactory explanations have been advanced for this "alarming tendency to chronicity". ${ }^{10}$

Most of the articles concerning CFS assume a simple model in which an external agent, usually a virus, causes a fatiguing illness by persistent viral infection. ${ }^{11}$ immune dysfunction $^{12}$ or a mixture of the two. ${ }^{1314}$ We have argued that a more complex model is needed to explain the peculiar clinical features of this illness. ${ }^{1516}$

Recently attention has been given to the role of cognitive distortions, and the consequent maladaptive behaviour, as mediators of disability in many illnesses, both physical and psychological. ${ }^{17}$ Looking specifically at CFS, it is plausible that an initial infective trigger may begin a cycle in which both attributional and cognitive factors fuel avoidant behaviour. The initial symptoms, in particular fatigue and myalgia, engender a state of "learned helplessness", being potent, aversive and uncontrollable, ${ }^{18}$ and may also trigger or exacerbate the mood disorder that is found in many patients. ${ }^{19}$ Continuing attribution of all symptoms to a persistent, untreatable "virus", continues to increase helplessness, although preserves self esteem. ${ }^{18}$ Avoidant behaviour (which is reinforced by the advice currently offered to patients) sustains symptoms, by decreasing activity tolerance and increasing 
sensitivity to any stimulation, as does associated mood disorder. Re-exposure to activity causes more symptoms, ${ }^{20}$ and more fear. The result is a vicious circle of symptoms, avoidance, fatigue, demoralisation and depression-the clinical picture of CFS. A similar role for avoidance in perpetuating symptoms has been implicated in chronic pain. ${ }^{2122}$

Such a model implies treatments that differ from those suggested by a simple external agent/disease model. In particular, improvement should result from attention to mood disorder, cognitive distortions and finally behavioural avoidance. Instead of antiviral agents, therapeutic success should result from reducing avoidant behaviour, decreasing the perception of helplessness and improving mood, ${ }^{17}$ all of which are already an established part of the treatment of both chronic pain and fibromyalgia ${ }^{23}$ (the latter being closely related to $\mathrm{CFS}^{25}$ ). In practice this can involve using the techniques of cognitive behaviour therapy. ${ }^{26}$

We report the results of an open trial of cognitive behaviour therapy and antidepressants in patients who fulfilled the operational criteria for the syndrome and were referred for a neurological assessment of fatigue to the National Hospital for Neurology and Neurosurgery. ${ }^{19}$

\section{Methods}

Most of the sample reported in this paper has been described in detail in a previous paper. ${ }^{19} \mathrm{It}$ consists of fifty patients referred to our hospital for a medical assessment of severe fatigue for whom no neurological explanation could be found and conventional neurological tests were normal. The only difference is that the first 13 patients in the previous communication were not offered treatment, but have been replaced by 16 new patients. There are no clinical differences between the previous sample and the current extended sample.

As an entry criterion all patients had a principle complaint of physical fatigue. In addition 49/50 reported physical fatigability (that is, fatigue made worse by physical exertion), and 45/50 reported mental fatigue and fatigability. In addition to fatigue, $42(84 \%)$ complained of muscle pain, $43(86 \%)$ complained of post exercise myalgia. Over $50 \%$ of the sample also complained of the following symptoms: nausea, dizziness, daytime drowsiness, back pain, headaches, tremor, perspiration, eyestrain, paraesthesiae and insomnia. Three quarters $(76 \%)$ reported that their illness had begun after a "viral" infection, and $42 / 50(84 \%)$ believed they had postviral fatigue syndrome or "ME." Twenty seven (54\%) were members of the ME Association. Most had made their own diagnosis or had been diagnosed by a doctor specialising in "ME".

All patients fulfilling the criteria were referred by the neurological staff as part of a previous study into the nature of fatigue in the postviral syndrome. After completing self report questionnaires and a standardised interview ${ }^{19}$ all were given a brief description of treatment by the psychiatrists. Those who fulfilled operational criteria for depression were also started on antidepressant medication (see later). A further assessment took place between four to six weeks later, and was carried out by the two therapists. After further discussion an offer of treatment was made, and therapy started on the next visit.

Thirty two patients accepted the offer of treatment, of whom 26 were treated as outpatients. The mean therapist time was 7.5 hours, with a range from two to 20 hours of treatment. Six had șuch severe disability (being confined to bed or a wheelchair for most or all of the time), that it was necessary to admit them to hospital for a period of between three to eight weeks.

Treatment followed cognitive behavioural principles, ${ }^{31}$ derived from work on the management of chronic pain, ${ }^{27}$ but adapted for the particular problems of CFS. Full details and techniques are reported elsewhere, ${ }^{17}$ but a brief account follows. After a detailed assessment, patient and therapist identified prominent disabilities, and looked at the associations and reasons advanced for such disabilities. Cognitive distortions, when present, were noted, and patients were encouraged to look for alternative explanations. Self monitoring of both behaviour and cognitions was also encouraged. Behavioural targets were jointly agreed upon, based on avoided activities which the patient wished to resume. These were practised between sessions. The intention was to introduce predictability into the pattern of rest and activity, and to encourage self efficacy-this was phrased as "helping the patient, and not ' $M E$ ', to be in control". Later in treatment the patient was encouraged to break the association between symptoms and ceasing activity, so that sensitisation to symptoms and activity was replaced by tolerance.

Twenty patients were offered tricyclic antidepressants at the initial psychiatric interview. Dothiepin, a conventional tricyclic antidepressant, was prescribed in doses ranging from 75 to $150 \mathrm{mg}$, depending upon ability to tolerate side effects. All fulfilled the Research Diagnostic Criteria ${ }^{28}$ for probable or definite major depression. Three of those offered treatment declined. Similar treatment was also given to an additional three patients who fulfilled criteria for other psychiatric disorders (two somatisation, one anxiety), and were already receiving antidepressants. Those given antidepressants scored higher on scales measuring psychiatric symptoms, somatic symptoms, and mental fatigue, and were more functionally impaired. However, there were no differences in the length of illness, nor physical fatigue, which is consistent with previous observations on this sample. ${ }^{19}$

After four to six weeks all patients were seen by the behaviour therapists, at which time mood disorder was reassessed with the Beck Depression Inventory (BDI). ${ }^{29}$ There were no longer differences in the severity of mood disorder between those who had been prescribed antidepressants and those who had not 
(mean Beck Depression Score for those receiving antidepressants was $10 \cdot 2(6 \cdot 7-13 \cdot 8)$, compared with $8 \cdot 8(4 \cdot 1-13 \cdot 5)$, although they still had significantly higher numbers of somatic symptoms.

Two different sets of measures were used. The first comprised well validated self administered questionnaires recording psychiatric symptomatology. These were the 12 Item General Health Questionnaire (GHQ), ${ }^{30}$ the Hospital Anxiety and Depression Scale (HAD), ${ }^{31}$ Modified Somatic Discomforts Questionnaire, ${ }^{32}$ the Fear Questionnaire, ${ }^{33}$ the Beck Depression Inventory, ${ }^{29}$ and a new scale for recording physical and mental fatigue..$^{22} \mathrm{All}$ measures were completed between the neurological and psychiatric assessments, the exceptions were the Beck Depression Inventory and the Fear Questionnaire, which were given at the start of therapy, and thus are only available for those in treatment.

The second set of instruments recorded functional disability. The principal measure of disability was a self assessment of functional impairment. ${ }^{33}$ This consists of four visual analogue scales covering ability to work, home management, social and private leisure activities. Respondents are asked how much their problems have affected each of the designated areas. Ratings range from 0 ("not at all") to 8 ("very severe, I cannot do it"). The scores of the four visual analogue ratings of functional impairment were summed and treated as a single variable, labelled "impairment".

The second measure was of "problems and targets". ${ }^{37}$ The patient with the therapist identifies which of their problems is the most incapacitating, together with the associated cognition. Examples include "inability to resume my job because I always feel tired", or "I cannot get out of bed because of the pain". The appropriate target is then agreed by patient and therapist as something to work towards: it may be "to resume full time work", or "to be able to feed and dress myself unaided".

At the end of treatment all self report measures were repeated, usually being completed at home, although the behavioural measures of problems and targets were completed with the therapist. A five point self rated outcome scale was also completed, in which patients were asked to rate themselves along a scale from much better to much worse. As the aim of the therapy was to improve the management of symptoms, the end point of treatment was therefore when the patient was able to deal with symptoms without further therapist contact, or when the therapist felt he/ she had no more to offer. It was not when the patient had become asymptomatic, as many continued to improve at follow up.

Parametric statistics were used where appropriate, either on the initial variable or following $\log$ transformation (length). Non parametric statistics were used for the results of self report of functional impairment, problems, targets and attitudes. All means are quoted with $95 \%$ confidence intervals, and all significance testing is two tailed.

\section{Results}

Changes were noted in all aspects of life (table 1): social, private, work and leisure (table 2), as well as psychological symptoms (table 3 ). Of those who completed treatment, there was an overall self rated percentage improvement in disability of $60 \%$ (95\% confidence interval 41$78 \%$ ). In most, improvement occurred globally. The decline in functional disability correlated with a decrease in mood and anxiety related symptoms and also somatic symptoms (data not shown). Some experienced a greater than $50 \%$ improvement in symptoms and disability, but remained symptomatic, especially with a variety of somatic discomforts. In only three patients was there greater than a $50 \%$ decrease in psychiatric symptomatology without an equivalent improvement in functional impairment.

Twenty three $(70 \%$ of those starting therapy) patients described themselves as "better" or "much better". Stricter criteria, however, were used to determine complete recovery. These were as follows: below threshold scores on all of the GHQ, HAD and BDI measures; a score of 0 or 1 on both the physical fatigue scale (range $0-16$ ) and the mental fatigue scale (Range $0-10$ ), a global score of 1 ("much better"), and a functional disability score of 8 or below (range $0-32$ ). Nine patients met all these requirements $(28 \%)$.

Eighteen patients (36\%) declined the offer of treatment (11 when interviewed by the psychiatrist, seven by the behaviour therapist). This contrasts with only 5/50 refusals among the last 50 patients referred by neurologists for cognitive behaviour therapy for conditions other than CFS. Length of illness, presence of muscle pain, severity of mental or physical fatigue, history of "viral" illness, current psychiatric illness (RDC criteria) and functional impairment were not associated with the decision to accept or reject treatment. There was a trend for those refusing to be female (15/34 females refused compared to $3 / 16$ males; Chi squared $=2.03 ; p=0.15)$. There was also a trend for refusers to have lower scores on the GHQ-12 (mean GHQ score for refusers = 5.37 (3.39-7.35), accepted $=7 \cdot 59(6 \cdot 27-8 \cdot 9)$, $\mathrm{t}=1 \cdot 83, \mathrm{df}=46, \mathrm{p}=0.078)$. Although this did not reach conventional statistical significance, this may have been due to lack of power, since a similar non significant trend was observed for HAD scores. Attribution of symptoms also played a role. There was a trend for those refusing treatment to be more likely to believe their illness was entirely due to physical factors (Chi squared $=4 \cdot 61, \quad \mathrm{df}=2$, $\mathrm{p}=0.09$ ).

Five patients withdrew after starting the programme. Two had already been unable to

Table 1 Outcome of treatment

\begin{tabular}{lll}
\hline Global ratings & Completed & Drop out \\
\hline Much better & 16 & 0 \\
Better & 6 & 1 \\
About the same & 4 & 2 \\
Worse & 0 & 0 \\
Much worse & 1 & 2 \\
Total & 27 & 5 \\
\hline
\end{tabular}


Table 2 Changes in functional disability in those completing treatment $(\mathrm{NR}=27)$

\begin{tabular}{|c|c|c|}
\hline & $\begin{array}{l}\text { Individual scores ( } m \\
\text { Range } 0-8 . \\
\text { Before }\end{array}$ & After treatment \\
\hline $\begin{array}{l}\text { Ability to work } \\
\text { Home management } \\
\text { Social leisure activities } \\
\text { Private leisure activities } \\
\text { Total functional impairment } \\
\text { Principal target } \\
\text { Principal problem }\end{array}$ & $\begin{array}{c}6 \cdot 31(5 \cdot 57-7 \cdot 05) \\
5 \cdot 69(5 \cdot 04-6 \cdot 34) \\
5 \cdot 72(5 \cdot 08-6 \cdot 35) \\
5 \cdot 19(4 \cdot 52-5 \cdot 86) \\
22 \cdot 61(20 \cdot 42-24 \cdot 8) \\
6 \cdot 63(5 \cdot 94-7 \cdot 32) \\
6 \cdot 57(5 \cdot 99-7 \cdot 15)\end{array}$ & $\begin{array}{l}2.72(1.77-3.65)^{\star} \\
1.54(0.78-2 \cdot 17)^{\star} \\
2.08(1 \cdot 17-2.99)^{\star} \\
1.65(0.89-2 \cdot 41)^{\star} \\
7.96(4.92-11.00)^{\star} \\
1.52(0.87-2 \cdot 17)^{\star} \\
1.61(1.11-2 \cdot 13)^{\star}\end{array}$ \\
\hline
\end{tabular}

*Wilcoxon Matched-pairs signed ranks test: $P<0.001$

Table 3 Changes in symptoms

\begin{tabular}{|c|c|c|}
\hline & \multicolumn{2}{|c|}{ Individual scores (mean, $95 \% \mathrm{CI}$ ) } \\
\hline $\begin{array}{l}\text { GHQ-12 score: } \\
\text { Mental fatigue } \\
\text { Physical fatigue } \\
\text { HAD score } \\
\text { Somatic symptoms } \\
\text { Beck depression inventory } \\
\text { Fear questionnaire }\end{array}$ & $\begin{array}{c}7 \cdot 7(5 \cdot 7-9 \cdot 04) \\
5 \cdot 82(4 \cdot 82-6 \cdot 82) \\
12 \cdot 48(11 \cdot 16-13 \cdot 8) \\
17.91(14 \cdot 59-21 \cdot 23) \\
14 \cdot 33(12 \cdot 61-16 \cdot 65) \\
9 \cdot 79(7 \cdot 2-12 \cdot 37) \\
27 \cdot 93(20 \cdot 35-35 \cdot 51)\end{array}$ & 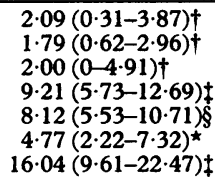 \\
\hline
\end{tabular}

*Wilcoxon Matched-pairs signed ranks test: $P<0.01$

+Wilcoxon Matched-pairs signed ranks test: $P<0.001$

tPaired t test $(\mathrm{df}=22), \mathrm{p}=0.014$

+ Paired t test $(\mathrm{df}=22), \mathrm{p}=0.014$
$\$$ Paired $\mathrm{t}$ test $(\mathrm{df}=26), \mathrm{p}<0.001$
Table 4 Influence of symptom attribution on treatment outcome

\begin{tabular}{lll}
\hline & $\begin{array}{l}\text { Physical (7) or } \\
\text { mainly physical } \\
(16)\end{array}$ & $\begin{array}{l}\text { Both (6) or } \\
\text { mainly } \\
\text { psychological (2) }\end{array}$ \\
\hline $\begin{array}{l}\text { Good outcome } \\
\text { Poor outcome }\end{array}$ & 14 & 8 \\
\hline $\mathrm{P}=0.04$ & 9 & 0 \\
$(1$ missing value $)$ & &
\end{tabular}

treatment Beck Depression Score in the good outcome group was $8 \cdot 25(5 \cdot 6-10 \cdot 9)$, compared with $11.66(5 \cdot 1-18 \cdot 2)$ in the poor outcome group; Mean HAD scores were 17.61 (13.2$22 \cdot 0)$ compared with $16 \cdot 11(10 \cdot 0-22 \cdot 2)$ ). However, $3 / 3$ patients who deteriorated were cases of affective disorder, as were $4 / 6$ of those unchanged. The second variable associated with a poor outcome was the strength of attribution to a physical cause (table 4). Of the seven patients who attributed their illness to exclusively physical causes six had a poor outcome. Finally, the role of the VP-1 antigen ${ }^{11}$ remains unclear. There was a trend for patients positive for this group specific enteroviral antigen to do worse, although this did not reach conventional statistical significance (Fisher's exact $=0.08)$. VP-1 antigen status was not obtained on eight cases.

Of the twenty three patients eligible to receive antidepressants, $16 / 23(69 \%)$ had a good outcome. Of the seven with a poor outcome, only one had received $150 \mathrm{mg}$ of dothiepin for six weeks. Of the other six, four had not tolerated antidepressants (defined as being unable to take a tricyclic antidepressant for more than seven days), and two had declined to take them. Only one of the three drug refusers therefore had a good outcome. The nine remaining patients who agreed to treatment did not fulfil the criteria for major depression, and received no antidepressants; $7 / 9(77 \%)$ had a good outcome.

All patients who successfully completed therapy have now been followed up for three months, as have $3 / 9$ with poor outcomes. Of the nine patients who finished symptom free, eight remain so. One has re-experienced some symptoms, but remains able to work. Most of the remainder are stable, and have maintained their improvement (four have continued to improve further of whom one is now symptom free). Three have experienced a gradual worsening of symptoms, but without any change in functional disability (two were associated with discontinuing antidepressants). In conclusion, at three months follow up the 23 who improved during the treatment all remain better than before.

\section{Discussion}

We report an uncontrolled pilot study of treatment of the chronic fatigue syndrome. The management of these patients followed our standard clinical management. The study is thus subject to all the deficiencies of a non blind, non randomised study. In the current climate of opinion, however, these results are of interest for several reasons. 
First, we have shown that something can be done for patients suffering from CFS and related syndromes. Most of those entering the trial experienced an improvement in symptoms and functional activity, and many were able to resume their occupations, or if not employed, experienced a return to previous levels of functioning.

Second, this study suggests that the view that little can be done for patients with CFS is inaccurate. In particular, we have shown that even those experiencing longer than two years of illness, and severe and persistent disability, can still return to premorbid functioning.

Third, the advice customarily given to those with illnesses of long duration, to avoid physical and mental activity, may not only be unnecessary, but sometimes inadvertently counterproductive. Much of the current self help literature and media reporting state that the approach we favour should lead to persistent illness and increased morbidity, especially in those with chronic disability.

Overall, only three out of 32 patients were worse after entering the trial. All had mood disorder on entry, which persisted throughout treatment. Two of the three were unable to tolerate conventional therapy in the form of antidepressants. A major reason for a lack of improvement was therefore persistent mood disorder. It is possible that others who would have suffered adverse effects declined the offer of treatment, and such fears were indeed the principal reason expressed for refusal. The accuracy of that perception is impossible to determine, but there were few clinical or symptomatic factors associated with refusal. Instead, the trend for those refusing treatment are more likely to attribute all their symptoms to a purely physical problem, and less likely to accept an interaction between physical and psychological, may reflect the external social pressures that complicate so much of CFS. ${ }^{34}$

Both persistent mood disorder and the strength of attributions of illness contributed to poor outcome. More tentatively, there was also a trend for an association between poor outcome and the persistence of enteroviral antigen. This may have been due to confounding factors, as the antigen is found in both depression ${ }^{35}$ and neurological disorders. ${ }^{36}$

As in any non blind, uncontrolled study the results must be interpreted cautiously. The observed improvements were, however, unlikely to be due to chance alone. The average duration of illness before referral was just under four years, after which time most authors state that little or no spontaneous recovery can be expected. ${ }^{237}$ It is not possible to determine the precise cause of improvement, nor to separate out the effects of antidepressant medication and cognitive behaviour therapy. It seems unlikely that improvements were solely due to medication, since a similar proportion of those not prescribed antidepressants achieved as good an outcome as those receiving medication. We also doubt that improvement was solely due to the physiological benefits of increased exercise, ${ }^{38}$ since behavioural targets were chosen on the basis of avoidance, not because of their physiological or ergonomic properties, which in practice were often minimal. It is not even possible to rule out the influence of non specific factors, such as therapist time and interest.

Demonstrating the benefits of cognitive behaviour therapy sheds little light on the nature of the initial pathology in CFS. The balance between physical, psychological and social factors changes over time in many illnesses. Our results are compatible with current theories on either a viral or immune precipitant. Such factors are not, however, the only cause of long term disability. Even if all cases of CFS are initially the result of viral infection, which seems unlikely, ${ }^{3}$ the secondary consequences, both social and psychological, may be a more potent cause of long term disability, analogous to the long term outcome of chronic pain ${ }^{2122}$ and head injury. ${ }^{39}$ Fortunately, such secondary factors are more amenable to treatment at present.

Details of the clinical characteristics of the patients confirm that the current sample was typical of hospital samples currently viewed as having severe postviral fatigue/"ME", and resembled those reported from other specialised referral centres. All fulfilled recent consensus criteria for CFS. ${ }^{40}$ Our findings can therefore be generalised to other CFS patients seen in hospital practice with severe fatigue of long duration and a diagnois of postviral fatigue syndrome or its equivalent. The results, however, cannot be assumed to apply to those seen in general practice, or with shorter durations of illness.

Future trials are essential to clarify these results. In particular, randomisation of patients to either the above therapy, rest or antidepressants is necessary. Other improvements, including independent ratings, should be used, although any trial of this nature will ultimately depend upon self report. Finally, it is hoped that the current unwelcome "polarisation of attitudes"10 concerning the nature of CFS will change, to allow empirical treatments, as outlined in this paper, to become more widely available than at present.

In conclusion, these results confirm the intuition of Jerome Frank, ${ }^{41}$ who wrote that "Patients suffering from unfamiliar diseases tend to develop emotional reactions which impede recovery, such as anxiety, resentment and confusion. To keep disability at a minimum, therapeutic efforts must be directed not only to overcoming the pathogenic agent but to maintaining the patient's confidence in the physician, and encouraging his expectation of a return to useful activity".

We thank the medical and nursing staff of the National Hospital for their continuing support. Most of all we thank the patients who participated in the trial, often under trying circumstances. SW is supported by a Wellcome Training Fellowship in Epidemiology, MR is partly supported by the SCARFE Trust.

1 David A, Wessely S, Pelosi A. Post-viral Fatigue: Time for a New Approach. Br Med J 1988;296:696-9.

2 Smith D. Understanding ME. London: Robinson, 1989.

3 Wessely S, Thomas PK. Chronic fatigue syndrome (myalgic Wessely $\mathrm{S}$, Thomas $\mathrm{PK}$. Chronic fatigue syndrome (myalgic
encephalomyelitis or postviral fatigue). In: Kennard C, ed. Recent advances in neurology, Vol 6. Edinburgh Churchill Livingstone, 1990: 85-131. 
4 Holmes G, Kaplan J, Gantz N. et al. Chronic Fatigue Syndrome: A Working Case Definition. Annals Int Med 1988;108:387-9.

5 Swartz $M$. The chronic fatigue syndrome-one entity or many? New Eng J Med 1988;319:1726-8.

6 Behan P, Behan W. The Postviral Fatigue Syndrome, $C R C$ Critical Reviews in Neurobiology 1988;42:157-8.

7 Straus S, Dale J, Tobi M, et al. Acyclovir treatment of the chronic fatigue syndrome: lack of efficacy in a placebocontrolled trial. New Eng $J$ Med 1988;319:1692-8.

8 Bayliss R, op cit Dawson J. Brainstorming the postviral fatigue syndrome. $\mathrm{Br}$ Med J 1988;297:1151.

9 Wessely $S$. The natural history of fatigue and myalgia syndromes. In: Sartorius N, Goldberg D, de Girolamo G, Costa e Silva J, Lecrubier Y, Wittchen H, eds. Psychological disorders in general medical settings. Bern: Hans Huber, 1990:82-97.

10 Smith D. Myalgic encephalomyelitis. (Members Reference Book). $R$ Coll Gen Pract. London, Sabre Crown, 1989:247-50.

11 Yousef G, Bell E, Mann G, et al Chronic enterovirus infection in patients with postviral fatigue syndrome. Lancet 1988;i:146-50.

12 Lloyd A, Wakefield D, Boughton C, Dwyer J. Immunological Abnormalities in the Chronic Fatigue Syndrome. Med J Aust 1989;151:122-4.

13 Behan P, Behan W, Bell E. The postviral fatigue syndromean analysis of the findings in 50 cases. $J$ Infection 1985;10:211-22.

14 Komaroff A. Chronic Fatigue Syndromes: relationship to chronic viral infections. J Virological Methods 1988;21: 3-10.

15 Wessely S, David A, Butler S, Chalder T. The management of the chronic "post-viral" fatigue syndrome. J R Coll Gen Pract 1989;39.26-9.

16 Wessely S, Butler S, Chalder S, David A. The cognitive behavioural management of the postviral fatigue syndrome. In: Jenkins R, Mowbray J, eds. The postviral fatigue syndrome $(M E)$. Chichester: John Wiley, (in fatigue

17 Sensky T. Patients' reactions to illness. Brit Med J 1990;300:622-3.

18 Powell R, Dolan R, Wessely S. Attribution and self esteem in depression and chronic fatigue syndromes. J Psychosomatic Research, 1990;34:665-73.

19 Wessely S, Powell R. Fatigue Syndromes: A comparison of chronic "postviral" fatigue with neuromuscular and affective disorders. J Neurol Neurosurg Psychiatry 1989;52: 940-8.

20 Editorial. Aching Muscles after Exercise. Lancet 1987; ii: $1123-5$.
21 Keefe F, Gil K. Behavioural concepts in the analysis of chronic pain syndromes. J Consult Clinical Psychology 1986;54:776-83.

22 Philips $\mathrm{H}$. Avoidance behaviour and its role in sustaining chronic pain. Behav Res Therapy 1987;25:273-9.

23 McCain G, Bell D, Mai F, Holliday P. A controlled study of the effects of a supervised cardiovascular fitness training program on the manifestations of primary fibromylagia. Arthritis Rheum 1988;31:1135-41.

24 Yunus $M$. Diagnosis, etiology and management of fibromyalgia syndrome: an update Comprehensive Therapy 1988;14:8-20.

25 Goldenberg D. Fibromyalgia and other chronic fatigue syndromes: is there evidence for chronic viral disease? Seminars in Arthritis and Rheumatism 1988;18:111-20.

26 Hawton K, Salkovskis P, Kirk J, Clark D. Cognitive behaviour therapy for psychiatric problems: a practical guide. Oxford: Oxford University Press, 1989.

27 Pither C. Treatment of persistent pain. Br Med J 1989;299:1239.

28 Spitzer R, Endicott J, Robins E. Research diagnostic criteria (RDC) for a selected group of function disorders, 3rd ed. New York: New.York State Psychiatric Institute, 1977.

29 Beck A, Ward C, Mendelson M, Mock J, Erbaugh J. An inventory for measuring depression. Arch Gen Psychiatry 1961;4:561-71.

30 Goldberg $\mathrm{D}$. The detection of a psychiatric illness by questionnaire. London: Oxford University Press, 1972.

31 Zigmond A, Snaith R. The Hospital Anxiety and Depression Scale. Acta Psycha Scanda 1983;67:361-70.

32 Wittenborn J, Buhler R. Somatic Discomforts Among Depressed Women. Arch Gen Psychiatry 1979;36:465-71.

33 Marks I. Behavioural psychotherapy: Maudsley pocket book of clinical management. Bristol: Wright, 1986.

34 Wessely $S$. "Old wine in new bottles": neurasthenia and "ME". Psychological Medicine 1990;20:35-53.

35 Lynch S, Seth R. Postviral fatigue syndrome and the VP-1 antigen. Lancet 1989;ii:1160-1.

36 Halpin D, Wessely S. VP-1 Antigen in chronic postviral fatigue syndrome. Lancet 1989;i:1028-9.

37 Shepherd C. Living with ME: a self-help guide. London Heinemann, 1989.

38 Edwards RH. Muscle fatigue and pain. Acta Med Scand 1986;Suppl. 711:179-88.

39 Lishman A. Physiogenesis and psychogenesis in the postconcussional syndrome. Br J Psychiatry 1988;153:460-9.

40 Sharpe M, Archand L, Banatvala J, et al. Chronic fatigue syndrome: guidelines for research. J Roy Soc Med 1991, (in press).

41 Frank J. Emotional reactions of American soldiers to an unfamiliar disease. Am J Psychiatry 1946;144:631-40. 